\title{
ANGULAR DIFFERENTIAL CROSS-SECTIONS FOR ELECTRON TRANSFER IN COLLISIONS BETWEEN PROTON AND $\mathrm{He}^{+}(1 s)$ IONS
}

\author{
S. Bhattacharyya*, K. Pathak \\ Dept. of Physics, Gokhale Memorial Girls College, Calcutta-700020, India \\ A. BECKER AND F.H.M. FaISAL \\ Dept. of Theoretical Physics, Bielefeld University, Bielefeld, Germany \\ (Received February 14, 1996; revised version June 25, 1996; \\ in final form September 4, 1996) \\ Angular differential cross-sections for the formation of $\mathrm{H}$, as a result of \\ electron capture by proton from $\mathrm{He}^{+}(1 s)$ at the centre-of-mass energies from \\ 4 to $24 \mathrm{keV}$, are calculated using distorted wave Coulomb-Born approxima- \\ tion. The total interaction potentials in the prior-interaqtion channel and in \\ the post-interaction channel are considered. Coulomb boundary conditions \\ are taken care of with the proper choice of the perturbation potentials in \\ both the prior and the post channels. A prior-post discrepancy is noted. \\ Existing theoretical results are presented along with the present results.
}

PACS numbers: $34.70 .+e, 34.40 .+n$

\section{Introduction}

Charge transfer in collision between positive ions is of considerable theoretical and experimental interest in connection with their practical applications in laser physics, in plasma diagnostics, in research in thermonuclear fusion and to the properties of astrophysical objects.

In this paper we formulate an expression for angular distribution of $\mathrm{H}$ formed due to electron capture in collision between $\mathrm{H}^{+}$and hydrogen type ions. The result for

$$
\mathrm{H}^{+}+\mathrm{He}^{+}(1 s) \rightarrow \mathrm{H}(1 s)+\mathrm{He}^{2+}
$$

is presented for collision energy lying between $4-24 \mathrm{keV}$ in the centre of mass (CM) frame. The only other theoretical paper on the above process is by Winter [1]. An experiment on reaction (1) is in progress by the Giessen group [2] in Germany.

\footnotetext{
*Corresponding author's mailing address: 370/1, N.S.C. Bose Road, Calcutta-700047, India.
} 
We used Coulomb-Born approximation [3] with the total interaction potential, i.e. the proton $\left(\mathrm{H}^{+}\right)$interacting with both the bound electron and the nucleus of $\mathrm{He}^{1+}$. Eventually we took the exact form of the interaction potential. The boundary conditions [4] are taken care of for the post as well as for the prior forms of the interaction potentials. Momentum dependent phase factors enter into the interaction potentials in the initial and in the final channels. These phase factors can be associated with the translational factors arising out of the relative motions in charge transfer collisions.

Winter [1] has calculated the differential cross-section (DCS) for the process (1) in Eikonal approximations using both the triple-centre atomic-state basis and double-centre Sturmian pseudo-state basis. Significant quantitative differences were found at various energies. In the present case maximum contribution to the cross-section comes from the very small angular range $\left(0^{\circ}\right.$ to $\left.0.1^{\circ}\right)$ in the forward direction, but, unlike that by Winter, the present result decreases rapidly with angle. The present results with the post and the prior interaction potentials are shown graphically along with the results by Winter for CM-energies $4 \mathrm{keV}, 5 \mathrm{keV}$, $12.5 \mathrm{keV}$ and $14 \mathrm{keV}$. The present DCS with respect to the solid angle at $10 \mathrm{keV}$ is found to have similar type of variation with the CM-scattering angle $(\theta)$ as that obtained by the experimental group in Giessen [2] for the reaction

$$
\mathrm{He}^{+}+\mathrm{He}^{+} \rightarrow \mathrm{He}^{0}+\mathrm{He}^{2+},
$$

which is similar in principle to reaction (1). Differences are noted in DCS with the prior and the post interaction potentials. However, the discrepancies are very small for low angle scattering. The boundary conditions necessary for the asymptotic vanishing of the interaction potential at large distances are achieved in the present case.

\section{Mathematical formulation}

The charge transfer reaction under consideration is

$$
\mathrm{A}^{Z_{\mathrm{A}}^{+}}+\mathrm{B}^{\left(Z_{\mathrm{B}}-1\right)^{+}} \rightarrow \mathrm{A}^{\left(Z_{\mathrm{A}}-1\right)^{+}}+\mathrm{B}^{Z_{\mathrm{B}}^{+}},
$$

where the projectile $\mathrm{A}_{\mathrm{A}}^{Z^{+}}$is a bare ion and $\mathrm{B}^{\left(Z_{\mathrm{B}}-1\right)^{+}}$is the hydrogen type target ion. The coordinates in the laboratory system are shown in Fig. 1. $\boldsymbol{R}_{a}, \boldsymbol{R}_{b}$ and $r_{0}$ denote the coordinates of $\mathrm{A}^{Z_{\mathrm{A}}^{+}}, \mathrm{B}^{\left(Z_{\mathrm{B}}-1\right)^{+}}$and the electron e respectively. $C_{\mathrm{A}}$ and $C_{\mathrm{B}}$ are the coordinates of the $\mathrm{CM}$ of $\left(\mathrm{A}_{\mathrm{A}}^{Z^{+}}, \mathrm{e}\right)$ system and $\left(\mathrm{B}^{\left(Z_{\mathrm{B}}-1\right)^{+}}, \mathrm{e}\right)$ system respectively.

The total potential energy of the system is

$$
U=\frac{Z_{\mathrm{A}} Z_{\mathrm{B}}}{\left|\boldsymbol{R}_{\mathrm{A}}-\boldsymbol{R}_{\mathrm{B}}\right|}-\frac{Z_{\mathrm{A}}}{\left|\boldsymbol{R}_{\mathrm{A}}-\boldsymbol{r}_{0}\right|}-\frac{Z_{\mathrm{B}}}{\left|\boldsymbol{R}_{\mathrm{B}}-r_{0}\right|} \text {. }
$$

The operator expressions for the kinetic energies of relative motions of the interacting system for the initial and the final channels are respectively

$$
T_{\mathrm{i}}=-\frac{1}{2 \mu_{b}} \nabla_{\boldsymbol{r}_{b}}^{2}-\frac{1}{2 \mu_{\mathrm{i}}} \nabla_{\boldsymbol{\rho}_{\mathrm{i}}}^{2}-\frac{1}{2 M} \nabla_{\boldsymbol{R}}^{2}
$$




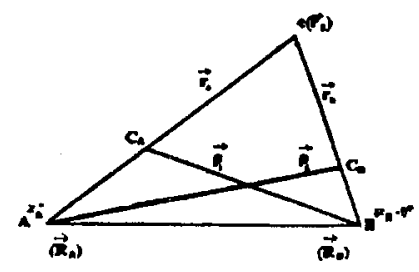

Fig. 1. Coordinate system: $\mathrm{A}^{Z_{\mathrm{A}}^{+}}, \mathrm{B}^{\left(Z_{\mathrm{B}}-1\right)^{+}}$and $\mathrm{e}^{-}$denote the bare ion of nuclear charge $Z_{\mathrm{A}}$, hydrogen type ion of nuclear charge $Z_{\mathrm{B}}$ and the electron, respectively. $C_{\mathrm{A}}$ is the centre of mass of the $\left(\mathrm{e}^{-}-\mathrm{A}^{z_{A}^{+}}\right)$system and $C_{B}$ is that of the $\left(\mathrm{e}^{--}-\mathrm{B}^{\left(Z_{B}-1\right)^{+}}\right)$system.

and

$$
T_{\mathrm{f}}=-\frac{1}{2 \mu_{a}} \nabla_{\boldsymbol{r}_{a}}^{2}-\frac{1}{2 \mu_{\mathrm{f}}} \nabla_{\boldsymbol{\rho}_{\mathrm{f}}}^{2}-\frac{1}{2 M} \nabla_{\boldsymbol{R}}^{2}
$$

where $\mu_{a}, \mu_{b}, \mu_{\mathrm{i}}$ and $\mu_{\mathrm{f}}$ are the reduced masses associated with the relative coordinates $r_{a}, r_{b}, \rho_{\mathrm{i}}$ and $\rho_{\mathrm{f}}$ respectively. $\boldsymbol{R}$ is the CM of the whole system.

$$
\begin{aligned}
& \rho_{\mathrm{i}}=\boldsymbol{R}_{\mathrm{A}}-\frac{M_{\mathrm{B}} \boldsymbol{R}_{\mathrm{B}}+m_{\mathrm{e}} \boldsymbol{r}_{0}}{M_{\mathrm{B}}+m_{\mathrm{e}}}, \quad \boldsymbol{R}=\frac{M \boldsymbol{R}_{\mathrm{A}}+M_{\mathrm{B}} \boldsymbol{R}_{\mathrm{B}}+m_{\mathrm{e}} r_{0}}{M_{\mathrm{A}}+M_{\mathrm{B}}+m_{\mathrm{e}}}, \\
& \boldsymbol{r}_{b}=\boldsymbol{R}_{\mathrm{B}}-\boldsymbol{r}_{0}, \quad M=M_{\mathrm{A}}+M_{\mathrm{B}}+m_{\mathrm{e}}, \\
& C_{1}^{\prime}=\frac{m_{\mathrm{e}}}{M_{\mathrm{B}}+m_{\mathrm{e}}}, \quad A_{2}=\frac{M_{\mathrm{B}}}{M_{\mathrm{B}}+m_{\mathrm{e}}} .
\end{aligned}
$$

In the relative and the centre-of-mass coordinates of the initial system, the potential energy $U$ in (4) becomes

$$
U_{\mathrm{i}}=\frac{Z_{\mathrm{A}} Z_{\mathrm{B}}}{\left|\rho_{\mathrm{i}}-C_{1}^{\prime} r_{b}\right|}-\frac{Z_{\mathrm{A}}}{\left|\rho_{\mathrm{i}}-A_{2} r_{b}\right|}-\frac{Z_{\mathrm{B}}}{\left|r_{b}\right|}
$$

Similarly, the expression for $U$ in the final channel is

$$
U_{\mathrm{f}}=\frac{Z_{\mathrm{A}} Z_{\mathrm{B}}}{\left|\rho_{\mathrm{f}}-C_{1} \boldsymbol{r}_{b}\right|}-\frac{Z_{\mathrm{A}}}{\left|\boldsymbol{r}_{a}\right|}-\frac{Z_{\mathrm{B}}}{\left|\rho_{\mathrm{f}}+B_{1} \boldsymbol{r}_{a}\right|}
$$

where

$$
\begin{aligned}
& \rho_{\mathrm{f}}=\boldsymbol{R}_{\mathrm{B}}-\frac{M_{\mathrm{A}} \boldsymbol{R}_{\mathrm{A}}+m_{\mathrm{e}} \boldsymbol{r}_{0}}{M_{\mathrm{A}}+m_{\mathrm{e}}}, \quad r_{a}=\boldsymbol{R}_{\mathrm{A}}-r_{0}, \\
& C_{1}=\frac{m_{\mathrm{e}}}{M_{\mathrm{A}}+m_{\mathrm{e}}}, \quad B_{1}=\frac{M_{\mathrm{A}}}{M_{\mathrm{A}}+m_{\mathrm{e}}} .
\end{aligned}
$$

The total Hamiltonian of the system, corresponding to the above two expressions, can be written as follows: 


$$
\begin{aligned}
& H=T_{\mathrm{i}}+U_{\mathrm{i}}=H_{0}^{\mathrm{i}}+V_{\mathrm{i}} \\
& =\left\{\left(-\frac{1}{2 \mu_{b}} \nabla_{r_{b}}^{2}-\frac{Z_{\mathrm{B}}}{\left|r_{b}\right|}\right)+\left[-\frac{1}{2 \mu_{\mathrm{i}}} \nabla_{\boldsymbol{\rho}_{\mathrm{i}}}^{2}+\frac{Z_{\mathrm{A}}\left(Z_{\mathrm{B}}-1\right)}{\left|\rho_{\mathrm{i}}\right|}\right]-\frac{1}{2 M} \nabla_{\boldsymbol{R}}^{2}\right\} \\
& +\left\{Z_{\mathrm{A}}\left(Z_{\mathrm{B}}-1\right)\left(\frac{1}{\left|\rho_{\mathrm{i}}-C_{1}^{\prime} \boldsymbol{r}_{b}\right|}-\frac{1}{\left|\rho_{\mathrm{i}}\right|}\right)+Z_{\mathrm{A}}\left(\frac{1}{\left|\rho_{\mathrm{i}}-C_{1}^{\prime} r_{b}\right|}-\frac{1}{\left|\rho_{\mathrm{i}}+A_{2} r_{b}\right|}\right)\right\} \text {. } \\
& H=T_{\mathrm{f}}+U_{\mathrm{f}}=H_{0}^{\mathrm{f}}+V_{\mathrm{f}} \\
& =\left\{\left(-\frac{1}{2 \mu_{a}} \nabla_{\boldsymbol{r}_{a}}^{2}-\frac{Z_{\mathrm{A}}}{\left|\boldsymbol{r}_{a}\right|}\right)+\left[-\frac{1}{2 \mu_{\mathrm{f}}} \nabla_{\boldsymbol{\rho}_{\mathrm{i}}}^{2}+\frac{Z_{\mathrm{B}}\left(Z_{\mathrm{A}}-1\right)}{\left|\boldsymbol{\rho}_{\mathrm{f}}\right|}\right]-\frac{1}{2 M} \nabla_{\boldsymbol{R}}^{2}\right\} \\
& +\left\{Z_{\mathrm{B}}\left(Z_{\mathrm{A}}-1\right)\left(\frac{1}{\left|\rho_{\mathrm{f}}-C_{1} \boldsymbol{r}_{a}\right|}-\frac{1}{\left|\rho_{\mathrm{f}}\right|}\right)+Z_{\mathrm{B}}\left(\frac{1}{\left|\rho_{\mathrm{f}}-C_{1} r_{a}\right|}-\frac{1}{\left|\rho_{\mathrm{f}}+B_{1} r_{a}\right|}\right)\right\} \text {. }
\end{aligned}
$$

Assuming the projectile as proton, $Z_{\mathrm{A}}=1$, and writing the interaction potentials $V$ in the prior and in the post form as $V_{\mathrm{i}}$ and $V_{\mathrm{f}}$ respectively, we obtain from (9) and (10)

$$
V_{\mathrm{i}}=\left(Z_{\mathrm{B}}-1\right)\left(\frac{1}{\left|\rho_{\mathrm{i}}-C_{1}^{\prime} r_{b}\right|}-\frac{1}{\left|\rho_{\mathrm{i}}\right|}\right)+\left(\frac{1}{\left|\rho_{\mathrm{i}}-C_{1}^{\prime} r_{b}\right|}-\frac{1}{\left|\rho_{\mathrm{i}}+A_{2} r_{b}\right|}\right)
$$

and

$$
V_{\mathrm{f}}=Z_{\mathrm{B}}\left(\frac{1}{\left|\rho_{\mathrm{f}}-C_{1} r_{a}\right|}-\frac{1}{\left|\rho_{\mathrm{f}}+B_{1} r_{a}\right|}\right)
$$

$V_{\mathrm{i}}$ and $V_{\mathrm{f}}$ vanish in the asymptotic limit and eventually satisfy the Coulomb boundary condition [4] necessary for charge transfer collisions.

Using Eqs. (6a) and (8a) we write $\rho_{\mathrm{f}}$ and $r_{a}$ in terms of $\rho_{\mathrm{i}}$ and $r_{b}$ and obtain

$$
\rho_{\mathrm{f}}=A_{1} r_{b}-B_{1} \rho_{\mathrm{i}}, \quad \boldsymbol{r}_{a}=\rho_{\mathrm{i}}+A_{2} r_{b},
$$

where

$$
A_{1}=\frac{m_{\mathrm{e}}\left(M_{\mathrm{A}}+M_{\mathrm{B}}+m_{\mathrm{e}}\right)}{\left(M_{\mathrm{A}}+m_{\mathrm{e}}\right)\left(M_{\mathrm{B}}+m_{\mathrm{e}}\right)} .
$$

Hence, from Eq. (12)

$$
V_{\mathrm{f}}=Z_{\mathrm{B}}\left(\frac{1}{\left|\rho_{\mathrm{i}}-C_{1}^{\prime} r_{b}\right|}-\frac{1}{\left|r_{b}\right|}\right) \text {. }
$$

Eigenfunctions of the channel Hamiltonian $H_{0}^{\mathrm{i}}$ and $H_{0}^{\mathrm{f}}$ as given by Fujiwara [5], are respectively

$$
\begin{aligned}
& \psi_{\mathrm{i}}=\phi_{\mathrm{i}}\left(r_{b}\right) \chi_{\mathrm{i}}\left(k_{\mathrm{i}}, \rho_{\mathrm{i}}\right), \\
& \psi_{\mathrm{f}}=\phi_{\mathrm{f}}\left(r_{a}\right) \chi_{\mathrm{f}}\left(k_{\mathrm{f}}, \rho_{\mathrm{f}}\right),
\end{aligned}
$$

where $\chi_{\mathrm{i}}$, the eigenfunction for relative motion of the colliding system in the initial channel, is expressed by Coulomb wave $F_{\mathrm{c}}\left(k_{\mathrm{i}}, \rho_{\mathrm{i}}\right)$, while, in the final channel, $\chi_{\mathrm{f}}$ is a plane wave. Here, $k_{\mathrm{i}}$ and $k_{\mathrm{f}}$ are respectively the relative momenta in the initial 
and final channels. The transition matrix element $V_{\mathrm{f}}^{\text {prior(post) }}$ of the prior (post) interaction $V_{\mathrm{i}}\left(V_{\mathrm{f}}\right)$ between the initial state $\psi_{\mathrm{i}}$ and the final state $\psi_{\mathrm{f}}$ is obtained in the Coulomb-Born approximation as follows. With the help of Eqs. (13), (15) and (16)

$$
\begin{aligned}
& V_{\mathrm{f}}^{\text {prior(post) }}=\int \psi_{\mathrm{f}} V_{\mathrm{i}, \mathrm{f}} \psi_{\mathrm{i}} \mathrm{d}^{3} \rho_{\mathrm{i}} \mathrm{d}^{3} r_{b} \\
& =\int \phi_{\mathrm{f}}^{*}\left(r_{a}\right) \chi_{\mathrm{f}}^{*}\left(k_{\mathrm{f}}, \rho_{\mathrm{f}}\right) V_{\mathrm{i}, \mathrm{f}} \phi_{\mathrm{i}}\left(r_{b}\right) F_{\mathrm{c}}\left(k_{\mathrm{i}}, \rho_{\mathrm{i}}\right) \mathrm{d}^{3} \rho_{\mathrm{i}} \mathrm{d}^{3} r_{b} .
\end{aligned}
$$

Using Taylor's expansion and writing $\dot{\boldsymbol{\rho}}_{\mathrm{i}}$ in terms of the momentum operator $-\mathrm{i} p$, we get for $V_{\mathrm{i}}$ and $V_{\mathrm{f}}$ (Eqs. (11) and (14)) the following expressions:

$$
\begin{aligned}
& V_{\mathrm{i}}=\frac{Z_{\mathrm{B}}-1}{\left|\rho_{\mathrm{i}}\right|}\left(\mathrm{e}^{-\mathrm{i} c_{1}^{\prime} \boldsymbol{r}_{b} \cdot \boldsymbol{p}}-1\right)+\frac{\mathrm{Z}_{\mathrm{A}}}{\left|\rho_{\mathrm{i}}\right|}\left(\mathrm{e}^{-\mathrm{i} c_{1}^{\prime} \boldsymbol{r}_{b} \cdot p}-\mathrm{e}^{-\mathrm{i} A_{2} r_{b} \cdot p}\right), \\
& V_{\mathrm{f}}=Z_{\mathrm{B}}\left(\frac{\mathrm{e}^{-\mathrm{i} c_{1}^{\prime} r_{b} \cdot p}}{\left|\rho_{\mathrm{i}}\right|}-\frac{1}{\left|r_{\mathrm{b}}\right|}\right) .
\end{aligned}
$$

We compute the DCS for charge transfer between proton and $\mathrm{He}^{+}(1 s)$ ion to produce hydrogen atom in the ground state. Taking $Z_{\mathrm{B}}=2$ the transition matrix element (Eq. (16)) with prior and post interaction potentials become, respectively

$$
\begin{aligned}
& V_{\mathrm{fi}}^{\text {prior }}=2 V_{\mathrm{fi}}(1)-V_{\mathrm{fi}}(2)-V_{\mathrm{fi}}(3), \\
& V_{\mathrm{fi}}^{\text {post }}=2 V_{\mathrm{fi}}(1)-2 V_{\mathrm{fi}}(4),
\end{aligned}
$$

where

$$
V_{\mathrm{f}}(n)=\int \phi_{\mathrm{f}}^{*}\left(r_{a}\right) \chi_{\mathrm{f}}^{*}\left(k_{\mathrm{f}}, \rho_{\mathrm{f}}\right) \frac{\mathrm{e}^{-\mathrm{i} s r_{b} \cdot p}}{\left|\rho_{\mathrm{i}}\right|} \phi_{\mathrm{i}}\left(r_{b}\right) \mathrm{F}_{\mathrm{c}}\left(k_{\mathrm{i}}, \rho_{\mathrm{i}}\right) \mathrm{d}^{3} \rho_{\mathrm{i}} \mathrm{d}^{3} r_{b}
$$

such that, for

$$
n=1, s=c_{1}^{\prime} ; n=2, s=0 ; \text { and } n=3, s=-A_{2}
$$

and

$$
V_{\mathrm{f}}(4)=\int \phi_{\mathrm{f}}^{*}\left(r_{a}\right) \chi_{\mathrm{f}}^{*}\left(k_{\mathrm{f}}, \rho_{\mathrm{f}}\right) \frac{1}{\left|r_{b}\right|} \phi_{\mathrm{i}}\left(r_{b}\right) \mathrm{F}_{\mathrm{c}}\left(k_{\mathrm{i}}, \rho_{\mathrm{i}}\right) \mathrm{d}^{3} r_{b} \mathrm{~d}^{3} \rho_{\mathrm{i}} .
$$

We use the transformations (13) for $r_{a}$ and $\rho_{\mathrm{f}}$ in computing $V_{\mathrm{f}}$. From space translation we obtain for the momentum operator $p$ in (18) and (19) the following equation $\left(\chi_{\mathrm{f}}\right.$ being the plane wave solution)

$$
\mathrm{e}^{-\mathrm{i} \lambda \cdot p_{p}}\left|\chi_{\mathrm{f}}^{*}\left(k_{\mathrm{f}}, \rho_{\mathrm{f}}\right)\right\rangle=\mathrm{e}^{-\mathrm{i} \lambda \cdot k_{\mathrm{f}}}\left|\chi_{\mathrm{f}}\left(k_{\mathrm{f}}, \rho_{\mathrm{f}}\right)\right\rangle,
$$

where

$$
\lambda=s r_{b} .
$$

After some lengthy calculations we obtain for $V_{\mathrm{f}}(n)$ and $V_{\mathrm{f}}(4)$ as shown in Appendix (Eqs. (9A), (11A)). 


\section{Results and discussion}

The charge transfer cross-section in the centre of mass coordinate becomes

$$
\sigma\left(E_{\mathrm{i}}\right)=\int \frac{\mu_{b}}{\left|k_{\mathrm{i}}\right|} \frac{\mathrm{d}^{3} k_{\mathrm{f}}}{2 \pi^{3}} 2 \pi\left|V_{\mathrm{f}}\right|^{2} \delta\left(E_{\mathrm{i}}-E_{\mathrm{f}}\right)=\int \frac{\mu_{b} \mu_{a}}{(2 \pi)^{3}}\left|\frac{k_{\mathrm{f}}}{k_{\mathrm{i}}}\right| 4 \pi^{2}\left|V_{\mathrm{fi}}\right|^{2} \sin \theta \mathrm{d} \theta
$$

We compute the differential cross-section with respect to the CM scattering angle $\theta$ and obtain

$$
\frac{\mathrm{d} \sigma(\theta)}{\mathrm{d} \theta}=\frac{\mu_{b} \mu_{a}}{(2 \pi)^{3}} 4 \pi^{2}\left|\frac{k_{\mathrm{f}}}{k_{\mathrm{i}}}\right|\left|V_{\mathrm{f}}\right|^{2} \sin \theta .
$$

In the charge transfer reaction (1), the energy and momentum transfers are very small. Eventually, the maximum contribution to the reaction comes from the small range of angles in the forward direction. We computed with both the post and the prior form of the interaction potentials. In the prior channel the interacting systems are charged, while in the post channel one of the interacting systems is neutral $(\mathrm{H}(1 s))$ while the other one is the fully stripped charged nucleus. With no target recoil in the projectile energy range under consideration, we find from (10A)

$$
\left|\delta_{1} / \delta_{2}\right| \ll 1
$$

and approximate $\phi(\lambda)=4 \pi / \delta_{1}$.
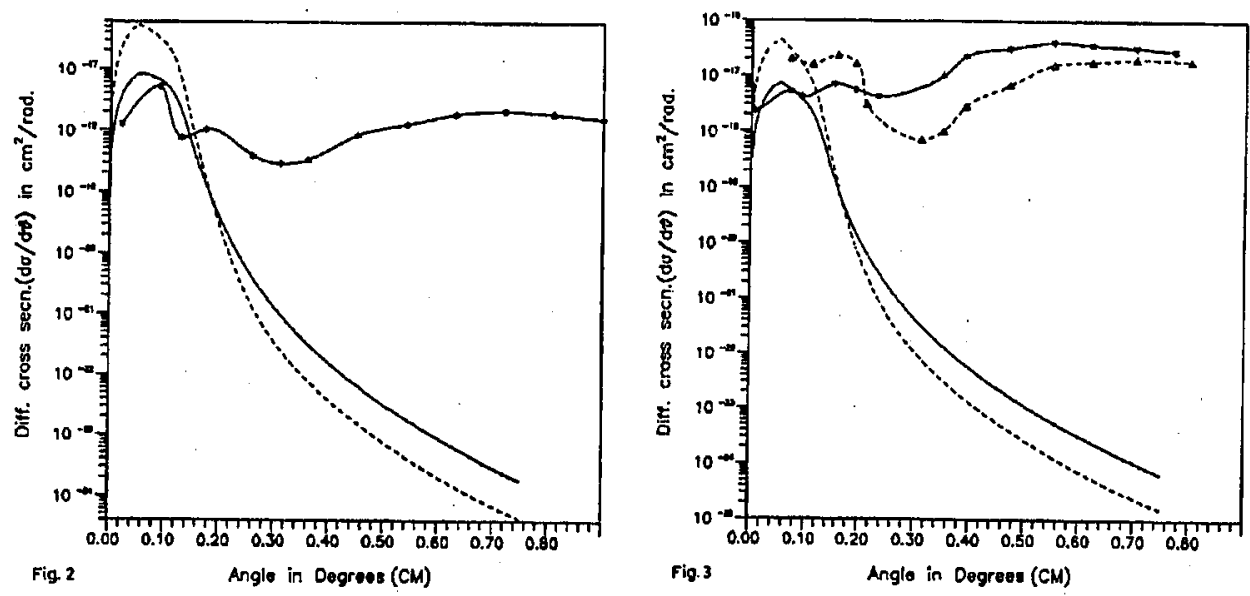

Fig. 2. Angular differential cross-section for charge transfer at $4 \mathrm{keV}$ CM-energy for the reaction: $\mathrm{H}^{+}+\mathrm{He}^{+}(1 s) \rightarrow \mathrm{H}(1 s)+\mathrm{He}^{2+}$. Present paper in: (i) prior interaction potential — , (ii) post interaction potential ..... . . Theoretical results by Winter [1] in Eikonal approximations using triple-centre, atomic-state approach -*-*--

Fig. 3 Angular differential cross-section for charge transfer at $5 \mathrm{keV}$ CM-energy. Present paper: the same as in Fig. 2. Theoretical results by Winter [1] in Eikonal approximation using: (i) triple-centre, atomic-state approach -*-*-, (ii) double-centre, Sturmian-pseudostate approach $--\triangle--\triangle-\cdot$. 

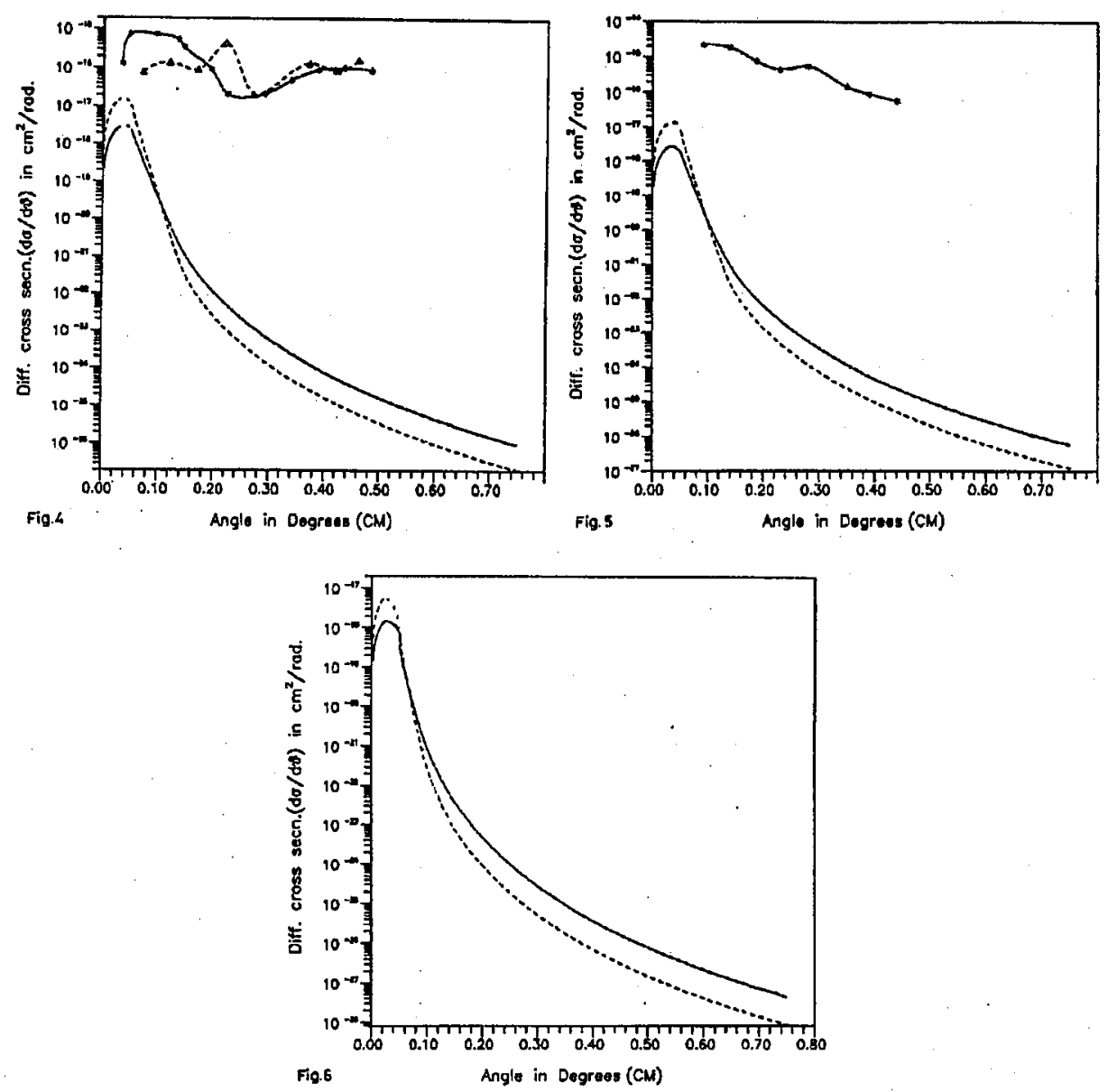

Fig. 4. The same as in Fig. 3 for $\mathrm{CM}$ collision energy $12.5 \mathrm{keV}$.

Fig. 5 The same as in Fig. 2 for CM-energy $14 \mathrm{keV}$.

Fig. 6 The same as in Fig. 2 for results at CM-energy $24 \mathrm{keV}$.

There is discrepancy, however small, between the post and the prior form of the cross-sections. This addresses to the question of non-orthogonality when the initial and the final wave functions (Eqs. (15), (16)) as given in Refs. [3] and [5], are used for the present problem. However, the discrepancies being small, the use of the mentioned wave functions in the present calculations is justified. The range of the angles giving maximum contribution is from $0^{\circ}$ to $0.1^{\circ}$ in both the prior and the post interaction potentials. As the angle increases to $0.5^{\circ}$, the DCS value falls by a factor of $10^{-5}$ from the value at $0.1^{\circ}$. At $4 \mathrm{keV}$ and $5 \mathrm{keV}$ CM-energies, Winter's results are comparable with those of the present results in the range $0^{\circ}$ to $0.1^{\circ}$. Beyond those angles, the present values for the DCS decrease exponentially, while results given by Winter maintain almost steady value with very little variation (Figs. 2-6). With the increase in the collision energies the 


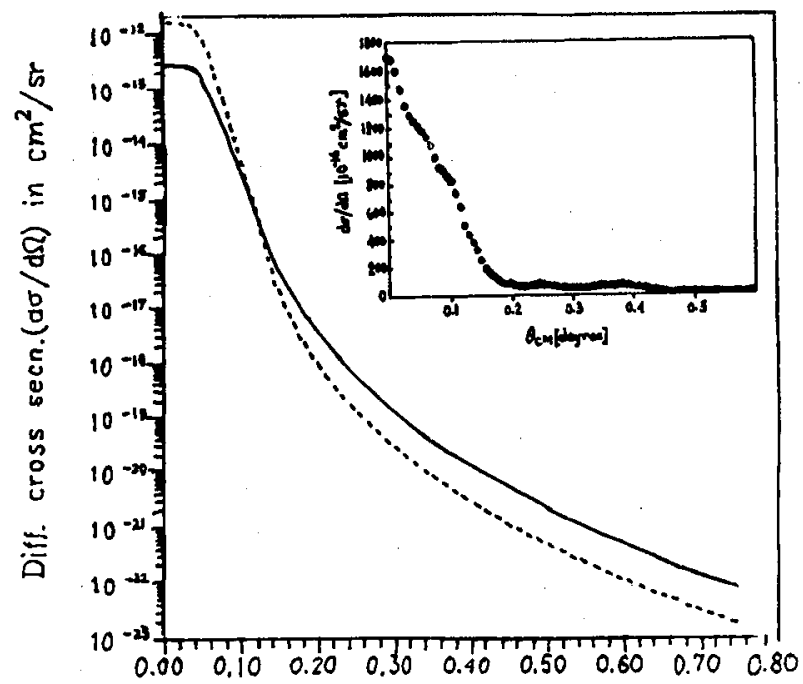

Fig. 7. The present results for $\mathrm{d} \sigma / \mathrm{d} \Omega$ as a function of CM-scattering angle at $\mathrm{CM}$-energy $10 \mathrm{keV}$. Inset in the figure shows the experimental result of Ref. [2] for the reaction: $\mathrm{He}^{+}+\mathrm{He}^{+} \rightarrow \mathrm{He}^{2+}+\mathrm{He}^{0}$ at $10 \mathrm{keV}$ CM-energy.

positions of the peaks shift towards the forward direction from $0.05^{\circ}$ at $4 \mathrm{keV}$ to $0.026^{\circ}$ at $24 \mathrm{keV}$, irrespective of the post and the prior interaction forms. Decrease in the peak values of the $\mathrm{d} \sigma / \mathrm{d} \theta$ are also noted with the increase in the CM-energy. To see the variation of the cross-sections with solid angle we computed the result at $10 \mathrm{keV}$ (Fig. 7). It is surprising to note that the behaviour like rapid drop of the cross-sections with solid angle, corroborates with the experimental results given by Giessen group (inset in Fig. 7), for a similar type of charge capture reaction [2]

$$
\mathrm{He}^{+}+\mathrm{He}^{+} \rightarrow \mathrm{He}^{+}+\mathrm{He}^{2+} \text {. }
$$

\section{Conclusion}

In this paper we have used distorted wave Coulomb-Born approximation with total interaction potential in the prior and post channels. The DCS has maxima close to the forward direction and decreases exponentially with angle. The small discrepancy in the DCS for the post and the prior interaction forms indicates the amount of non-orthogonality present in the wave functions as given in Refs. [3] and [5]. Eventually, to eliminate post-prior discrepancy, the exact solution of Schrödinger equation with the unperturbed Hamiltonians $H_{0}^{\mathrm{i}}$ and $H_{0}^{\mathfrak{f}}$ (Eqs. (9), (10)) is required. However, the discrepancy at small-angle scattering being small, one may use the wave functions by Fujiwara, without much loss of generality, for calculating the charge transfer reactions. The necessary boundary conditions, for asymptotic vanishing of the long range interaction potentials, are achieved. The 
differential cross-section $\mathrm{d} \sigma / \mathrm{d} \Omega$ plotted as a function of the CM-scattering angle at $10 \mathrm{keV}$ CM-energy, shows similar characteristic variation as obtained in the experiment [2] for $\mathrm{He}^{+}$capturing electron from $\mathrm{He}^{+}$. This depicts the validity of our theoretical approach in computing angular differential cross-section for electron capture in ion-ion collision. However, experimental results on the present problem are necessary before coming to a final conclusion regarding our theoretical approach.

\section{Appendix}

The detailed calculations for $V_{\mathrm{f}}(n)$ and $V_{\mathrm{f}}(4)$ are given below. The wave functions for $\mathrm{Ie}^{+}(1 s)$ ion in the initial channel and that of $\mathrm{H}(1 s)$ atom in the final channel are respectively

$$
\phi_{\mathrm{i}}\left(r_{b}\right)=N_{2} \mathrm{e}^{-\alpha_{2} r_{b}}, \quad \phi_{\mathrm{f}}\left(r_{a}\right)=N_{1} \mathrm{e}^{-\alpha_{1}\left|\rho_{\mathrm{i}}+A_{2} r_{\mathrm{b}}\right|} .
$$

$N_{1}$ and $N_{2}$ are the normalising factors for the wave functions of $\mathrm{H}(1 s)$ and $\mathrm{He}^{+}(1 s)$, respectively. The Coulomb wave function in the attractive field of the bound electron is

$$
F_{\mathrm{c}}=\mathrm{e}^{-\pi \eta / 2} \Gamma(1+\mathrm{i} \eta) \mathrm{e}^{-k_{\mathrm{i}} \cdot \rho_{\mathrm{i}}} F_{1}\left[-\mathrm{i} \eta, 1, \mathrm{i}\left(k_{\mathrm{i}} \rho_{\mathrm{i}}-k_{\mathrm{i}} \cdot \rho_{\mathrm{i}}\right)\right],
$$

where

$$
\eta=-\frac{M_{\mathrm{A}} M_{\mathrm{B}} /\left(M_{\mathrm{A}}+M_{\mathrm{B}}\right)}{\left|k_{\mathrm{i}}\right|} .
$$

The wave function for relative motion in the final channel

$$
\chi_{\mathrm{f}}\left(k_{\mathrm{f}}, \rho_{\mathrm{f}}\right)=(2 \pi)^{-3 / 2} \mathrm{e}^{-\mathrm{i} k_{\mathrm{i}} \cdot \rho_{\mathrm{i}}}
$$

where

$$
\rho_{\mathrm{f}}=A_{1} r_{b}-B_{1} \rho_{\mathrm{i}} .
$$

The integral $V_{\mathrm{fi}}(n)$, for $n=1$ to 3 , on using (25), (1A) and (3A), becomes

$$
\begin{aligned}
& V_{\mathrm{f}}(n)=\int N_{1} N_{2} \frac{1}{\left|\rho_{\mathrm{i}}\right|} \exp \left[-\alpha_{2} r_{b}-\alpha_{1}\left|\rho_{\mathrm{i}}+A_{2} r_{b}\right|\right. \\
& \left.\quad+\mathrm{i} k_{\mathrm{f}} \cdot\left(A_{1} r_{b}-B_{1} \rho_{\mathrm{i}}\right)-\mathrm{i} s r_{b} B_{1} k_{\mathrm{f}}\right] F_{\mathrm{c}}\left(k_{\mathrm{i}}, \rho_{\mathrm{i}}\right) \mathrm{d}^{3} \rho_{\mathrm{i}} \mathrm{d}^{3} r_{b} .
\end{aligned}
$$

The integration over $r_{b}$ in $(4 \mathrm{~A})$ is

$$
\begin{aligned}
I(n) & =\int \exp \left[-\alpha_{2} r_{b}-\alpha_{1}\left|A_{2} r_{b}+\rho_{\mathrm{i}}\right|+\mathrm{i}\left(A_{1}-B_{1} s\right) r_{b} \cdot k_{\mathrm{f}}\right] \mathrm{d}^{3} r_{b} \\
& =\frac{8 \pi \alpha_{1} \alpha_{2}}{\pi^{2}} I_{n}\left(\rho_{\mathrm{i}}\right),
\end{aligned}
$$

where $\int \exp \left(\mathrm{i} k \cdot \rho_{\mathrm{i}}\right)\left(\mathrm{d}^{3} k\right) /\left\{\left(\alpha_{1}^{2}+k^{2}\right)^{2}\left[\alpha_{2}^{2}+\left(A_{2} k+\left(A_{1}-s\right) k_{\mathrm{f}}\right)^{2}\right]^{2}\right\}=I_{n}\left(\rho_{\mathrm{i}}\right)$. Integration over $k$ in $I\left(\rho_{\mathrm{i}}\right)$ of $(5 \mathrm{~A})$ is done using Feynman identity

$$
\frac{1}{a^{2} b}=2 \int_{0}^{1} \frac{x \mathrm{~d} x}{[a x+b(1-x)]^{3}}
$$

and one obtains

$$
I_{n}\left(\rho_{\mathrm{i}}\right)=-\frac{1}{2 \alpha_{2}} \frac{\mathrm{d}}{\mathrm{d} \alpha_{2}} \iint_{0}^{1} \frac{2 x \mathrm{~d} x \exp \left(\mathrm{i} k \cdot \rho_{\mathrm{i}}\right) \mathrm{d}^{3} k}{\left[\left(k+t_{1}\right)^{2}+\lambda^{2}\right]^{3}\left[x+A_{2}^{2}(1-x)\right]^{3}},
$$


where

$$
\begin{aligned}
t_{1} & =\frac{\left(A_{1}-B_{1} s\right) A_{2} k_{\mathrm{f}}(1-x)}{x+A_{2}^{2}(1-x)} \\
h_{1} & =\frac{x\left[\alpha_{1}^{2}-\alpha_{2}^{2}-\left(A_{1}-B_{1} s\right)^{2} k_{\mathrm{f}}^{2}\right]+\alpha_{2}^{2}+\left(A_{1}-B_{1} s\right)^{2} k_{\mathrm{f}}^{2}}{x+A_{2}^{2}(1-x)} \\
\lambda^{2} & =-t_{1}^{2}+h_{1} .
\end{aligned}
$$

Using the integral formula

$$
\begin{aligned}
& \int \frac{\mathrm{e}^{\mathrm{i} t \cdot r_{\mathrm{d}} \mathrm{d}^{3} t}}{\left(\eta^{2}+t^{2}\right)^{3}}=-\frac{\pi^{2}}{4 \pi} \frac{\mathrm{d}}{\mathrm{d} \eta}\left(\frac{\mathrm{e}^{-\eta r}}{\eta}\right) \\
& I_{n}\left(\varrho_{\mathrm{i}}\right)=-\frac{1}{2 \alpha_{2}} \frac{\mathrm{d}}{\mathrm{d} \alpha_{2}} 2 \int_{0}^{1} \frac{x \mathrm{~d} x \mathrm{e}^{-\mathrm{i} t_{1} \cdot \rho_{\mathrm{i}}}}{\left[x+A_{2}^{2}(1-x)\right]^{3}}\left[-\frac{\pi^{2}}{4 \lambda} \frac{\mathrm{d}}{\mathrm{d} \lambda}\left(\frac{\mathrm{e}^{-\lambda \rho_{\mathrm{i}}}}{\lambda}\right)\right] .
\end{aligned}
$$

Using the above integrals we obtain

$$
\begin{aligned}
V_{\mathrm{f}}(n) & =\frac{N_{1} N_{2}}{(2 \pi)^{3 / 2}} \frac{8 \pi \alpha_{1} \alpha_{2}}{\pi^{2}}\left(-\frac{1}{2 \alpha_{2}} \frac{\mathrm{d}}{\mathrm{d} \alpha_{2}}\right) \int \mathrm{d}^{3} \rho_{\mathrm{i}} \int_{0}^{1} \frac{2 x \mathrm{~d} x}{\left[x+A_{2}^{2}(1-x)\right]^{3}}\left(-\frac{\pi^{2}}{4 \lambda}\right) \\
& \times(2 \pi)^{3 / 2} \frac{\mathrm{d}}{\mathrm{d} \lambda}\left[\frac{\exp \left(-\lambda \rho_{\mathrm{i}}\right)}{\lambda}\right] \frac{1}{\left|\rho_{\mathrm{i}}\right|} \exp \left(-\mathrm{i} t_{1} \cdot \rho_{\mathrm{i}}-\mathrm{i} B_{1} k_{\mathrm{f}} \cdot \rho_{\mathrm{i}}\right) F_{\mathrm{c}}\left(k_{\mathrm{i}}, \rho_{\mathrm{i}}\right) \\
= & G\left(-\frac{1}{2 \alpha_{2}} \frac{\mathrm{d}}{\mathrm{d} \alpha_{2}}\right) \int_{0}^{1} \frac{x \mathrm{~d} x}{\left[x+A_{2}^{2}(1-x)\right]^{3}}\left(-\frac{\pi^{2}}{4 \lambda}\right) \frac{\mathrm{d}}{\mathrm{d} \lambda}\left(\frac{\phi(\lambda)}{\lambda}\right),
\end{aligned}
$$

where

$$
\begin{aligned}
& G=2 \frac{N_{2} N_{1} 8 \pi \alpha_{2} \alpha_{1}}{\pi^{2}} \mathrm{e}^{-\pi \eta / 2} \Gamma(1+\mathrm{i} \eta), \\
& \phi(\lambda)=\int \mathrm{d}^{3} \rho_{\mathrm{i}} \frac{\mathrm{e}^{-\lambda \rho_{\mathrm{i}}}}{\left|\rho_{\mathrm{i}}\right|} \mathrm{e}^{-\mathrm{i}\left[\left(t_{1}+B k_{\mathrm{i}}\right) \cdot \rho_{\mathrm{i}}-k_{\mathrm{i}} \cdot \rho_{\mathrm{i}}\right]_{1} F_{1}\left(-\mathrm{i} \eta, 1,-\mathrm{i} k_{\mathrm{i}} \cdot \rho_{\mathrm{i}}+\mathrm{i} k_{\mathrm{i}} \rho_{\mathrm{i}}\right) .}
\end{aligned}
$$

Using Nordseik integral [6] we get

$$
\phi(\lambda)=\frac{4 \pi}{\delta_{1}}\left(1+\frac{\delta_{2}}{\delta_{1}}\right)^{\mathrm{i} \eta}
$$

where

$$
\delta_{1}=\lambda^{2}+L^{2}, \quad \delta_{2}=2\left[\left(t_{1}+B_{1} k_{\mathrm{f}}\right) \cdot k_{\mathrm{i}}-k_{\mathrm{i}}^{2}-\mathrm{i} \lambda k_{\mathrm{i}}\right]
$$

and

$$
L=t_{1}+B_{1} k_{\mathrm{f}}-k_{\mathrm{i}} .
$$

Similarly

$$
\begin{aligned}
& V_{\mathrm{f}}(4)=G^{\prime} \int \frac{x \mathrm{~d} x}{\left[x+A_{2}^{2}(1-x)\right]^{3}}\left(-\frac{\pi^{2}}{4 \lambda}\right) \frac{\mathrm{d}}{\mathrm{d} \lambda}\left[\frac{1}{\lambda} \frac{\mathrm{d}}{\mathrm{d} \lambda} \phi(\lambda)\right], \\
& G^{\prime}=2 \frac{4 \pi N_{1} N_{2}}{\pi^{2}} \mathrm{e}^{-\pi \eta / 2} \Gamma(1+\mathrm{i} \eta) .
\end{aligned}
$$

The integration over $X$ in (8A) and (11A) is carried out using a simple trapezoidal rule with a sufficiently small mesh $\Delta x$. 


\section{Acknowledgment}

One of the authors (S.B.) would like to thank DAAD, Bonn, for the financial support of the work during her stay in Germany. S.B. would like to thank Prof. N. Grün from the University of Giessen for helpful discussions, and Prof. E. Salzborn and Dr. F. Melchert from the Institute of Kernphysik, Giessen, for referring the problem to her.

S.B. and K.P. are thankful to UGC, New Delhi, for the grant No. F 10-100/90 (RBB-II), and to Sanchita Mitra for help in typing the manuscript.

\section{References}

[1] T.G. Winter, Phys. Rev. A 49, 1767 (1994).

[2] A. Pfeiffer, S. Krüdener, F. Melchert, L.P. Presnyakov, R. Schulze, S. Meuser, T. Bartsch, C. Brandau, K.v. Diemar, K. Huber, E. Salzborn, Nucl. Instrum. Methods Phys. Res. B 98, 240 (1995).

[3] S. Mukherjee, N.C. Sil, D. Basu, J. Phys. B 12, 1259 (1979).

[4] S. Mukherjee, D.P. Sural, J.F. McCann, I. Shimamura, Comments At. Mol. Phys. 28, 25 (1992).

[5] K. Fujiwara, J. Phys. Soc. Japan 41, 1350 (1976).

[6] A. Nordsieck, Phys. Rev. 93, 785 (1954). 